\title{
Perception of Affordances and Experience of Presence in Virtual Reality
}

\author{
Paweł Grabarczyk \\ Institute of Philosophy \\ University of Lodz \\ pagrab@gmail.com
}

\author{
Marek Pokropski \\ Institute of Philosophy \\ University of Warsaw \\ mpokropski@uw.edu.pl
}

\begin{abstract}
Recent developments in virtual reality technology raise a question about the experience of presence and immersion in virtual environments. What is immersion and what are the conditions for inducing the experience of virtual presence? In this paper, we argue that crucial determinants of presence are perception of affordances and sense of embodiment. In the first section of this paper, we define key concepts and introduce important distinctions such as immersion and presence. In the second and third sections, we respectively discuss presence, immersion and their determinants in detail. In the fourth and fifth sections, we argue for the importance of perception of affordances and sense of embodiment in increasing the degree of presence. Finally, we show the consequences of our view and discuss possible future implications
\end{abstract}

Keywords: cognitive science; phenomenology; perception; affordances; Virtual Reality.

My body is wherever there is something to be done (M. Merleau-Ponty, Phenomenology of Perception)

\section{Introduction. Some conceptual distinctions.}

The feeling of being present in the surrounding environment is possibly the most common and everyday experience for most people. However, this does not make it easier to explain. Presence studies have become more advanced since the emergence of VR technology, which to some extent induce can the experience of being present in another, virtual reality. In this paper, we try to explain the foundation of the experience of presence in virtual reality and how it can be supported by technological improvements. We argue that this phenomenon is essentially related to our sense of embodiment and possibility of action in the environment. Furthermore, we argue that one of the key fac- 
tors is perception of affordances. However, before we introduce our proposal, we would like to clarify and define some conceptual notions such as virtual reality, presence and immersion, which are interrelated yet different.

What is virtual reality (VR)? One definition proposes, "VR is any computermediated synthetic world that can be interactively experienced through sensory stimuli." (Fiore et al. 2009) 4 Two aspects of this definition that are worth pointing out (as they play an important role in our argumentation) is the direct reference to the technological medium and the "synthetic world". Another notable tradition of defining the notion of virtuality connects it with the notion of "simulation". Thus, "virtual" can be understood as simulated or surrogate environments or objects that elicit sensory responses (Hall et al., 2012). Two additional terms are sometimes used interchangeably with the notion of virtual reality: "virtual experience" and "virtual worlds". Additionally, people sometimes talk about "virtual environments" and "virtual objects". This provokes a host of conceptual questions: is every experience of a virtual world a virtual experience? How does the notion of virtual reality differ from the notion of virtual world? Is virtual reality simply a virtual world accessed through special gear (typically head mounted displays)? Is every act of watching digital media a type of "virtual experience"? If not, why does watching a screen becomes a "virtual experience" once I mount the screen on my head?

We do not have the ambition to define all of the notions listed above, but we can make more sense of them once we highlight some of their characteristic properties. Firstly, it is hard not to see that there is a relatively stable element to various uses of the term "virtual" that boils down to being a "nonphysical representation of something physical". This is what we mean when we talk about "virtual money" or "virtual maps". Note that the same intuition can be seen in cases in which the term "virtual" is used as a synonym of "potential": what we mean in these cases is that something physical is in fact only imaginary or conceptual. Thus, it might be best to think about virtual as a kind of a fundamental remediation of one ontological domain into different ontological domain. "Virtual" is thus very similar to the way we use the term "mental" or "imaginary". Not surprisingly, both these terms could be just as well used in the contexts presented above as we sometimes talk about imaginary worlds or imaginary experiences. One important factor that differentiates between virtual and imaginary (or mental) is that virtual objects, environments etc. can be shared: we can meet in a virtual room but not in an imaginary room. Even if we can both simultaneously imagine the same box, there is no way for me to rotate it in such a way that you could see its other side. Thus, building on Es-

\footnotetext{
${ }^{4}$ It should be pointed out that we use the term "virtual" as opposed to "physical" or "real", not as a synonym of "possible", "illusory" or "ideal" (Lister 2003). Obviously, this difference in meaning should not be understood as a simple equivocation. There are interesting philosophical dependencies between both senses of "virtual", but we do not cover them in this paper.
} 
pen Aarseth's definition (Aarseth 2001), we could define "virtual" as "nonphysical remediations of physical objects, spaces and processes that are accessible jointly to many agents".

So now-given this understanding of "virtual"-how can we make sense of expressions like "virtual reality", "virtual world" or "virtual experience"? Let us start with the idea of a virtual world. If we look at popular examples of virtual worlds discussed in the literature (Second Life being probably the most popular), you could see that it is compliant with the proposed definition of "virtual" as it comprises objects, people, processes, spaces etc. which are digital representations of physical counterparts. The main reason that we are inclined to call these digital constructs "worlds" is that they are persistent: they exist independently of the users who inhabit (or visit) them (Schroeder 1996, Schroeder 2008). This idea of persistency is one of the ways virtual worlds differ from virtual reality; the latter can just as well be a dreamlike constant flux of changing images and scenes without any noteworthy persistency.

The difference between ideas such as "virtual object", "virtual environment" and "virtual world" can be understood as just a difference of scale. Every virtual object exists in some virtual space; once the space is extended and can be traversed, it becomes a virtual environment. Moreover, a virtual environment populated with virtual objects can be understood as a virtual world. From the point of view of this paper, these differences in scale are not important. What is important is the difference between treating something as a mere representation (an image, an animation) and objectifying it; treating it as an object, environment or world.

As for the notion of "virtual experience", it may initially look to be very attractive, as it seems to save us from big ontological questions (because we do not have to talk about different worlds or realities). However, it in fact turns out to be very problematic. Firstly, it leads to an equivocation. Is a "virtual experience" an experience that is in itself virtual (whatever it might mean in this context), or simply an experience of something virtual? Some people might have philosophical reservations towards the first option that are, in fact, very Cartesian in nature. Can an experience be "virtual"? How does an experience of a virtual ball differ from the experience of a real ball? The difference lies on the side of the balls, not on the side of experiences they elicit. Is the whole point of $\mathrm{VR}^{5}$ not that the experiences it produces are indistinguishable from experiences of real things? Some philosophers might say that the fact they are indistinguishable does not mean they are the same. Therefore, once we start using the term "virtual experience" we quickly end up in the middle of a contemporary heated philosophical debate. This debate might be interesting in

\footnotetext{
${ }^{5}$ From this point we often abbreviate "virtual reality" to "VR"
} 
and of itself but is not crucial for the problem we wish to analyze in this paper. ${ }^{6}$ Thus, it is much better to choose the second option and say that virtual experience is an experience of something virtual (whether it be an object, an environment or a world).

Nevertheless, what about "virtual reality" as technology? Let us revise the conceptual distinctions we have made thus far. Firstly, we propose understanding the term "virtual" very broadly: as applicable to any shared nonphysical representation of physical reality (objects, environments or worlds). Secondly, we propose understanding the notion of virtual experience as simply the experience of these virtual representations. Now, we can understand the notion of "virtual reality" in a narrower way: as a computer-mediated way of eliciting a very specific type of virtual experience. Although VR technology was strongly dominated by the sense of sight in the initial phase of development, recently it aims to provide a fully multimodal experience. Contemporary VR researchers try to "incorporate" users into a virtual environment (VE) by giving him/her multimodal feedback: visual (HMD), tactile (gloves and vests), auditory and proprioceptive (tracking of bodily posture and movement). Thus according to Mel Slater we can think of VR as "sensory data generated by a computer system", which is perceived (via HMD or haptic interfaces) as "physical reality, especially when perception is enabled by use of the body in a manner similar to physical reality" (Slater 2009, p. 215). Such VR technology aims, so to speak, to re-embody the user into a virtual body and experientially transfer her into a virtual environment. This experience is most often characterized as "total immersion" or "presence", terms that became almost synonymous with virtual reality (especially in the popular discourse, but see (Steuer 1992) for an academic example of this).

Note that even though both terms can be easily criticized for being rather vague, this way of defining VR is much better than defining it via direct reference to head-mounted displays (or any other specific technological medium). Even though HMD's were (and still are) the dominant VR technology, there is nothing that precludes any other technology from becoming the new standard. For this reason, it is best not to tie general philosophical arguments directly to any given arbitrary technology. Additionally, as we argue in the two following sections, both terms can be explicated so they become useful for academic discussion.

The notions of presence and immersion are oftentimes used interchangeably. Presence can be very loosely described as a feeling of "being there" or a feeling of "being in the place one perceives". Immersion can be loosely described as a state of deep engagement in the medium (be it a book, a movie, video game or virtual environment). As pointed out in (Ermi \& Mäyrä 2005), the first

\footnotetext{
${ }^{6}$ See Burge 2005 for a good summary of this debate.
} 
is sometimes reserved for non-entertainment purposes (for example telemeetings or virtual museum tours, while the second mostly relates to video games. Another way to differentiate both can be found in (Witmer and Singer 1998), who acknowledge immersion, as well as involvement, as conditions of presence. Finally yet importantly, let us mention the perspective found in (Slater \& Wilbur 1997), where presence is reserved for the psychological feeling and immersion is reserved for the properties of artificial systems that facilitate the feeling of presence. One important advantage of this approach is that it does not conflate the psychological and technological aspects of the phenomenon of presence/immersion and allow us to study and measure it with different methods. For this reason, we employ Slater's/Wilbur's perspective in the current paper.

\section{Virtual presence}

In 1980, Marvin Minsky introduced the term "telepresence" (Minsky, 1980). Minsky considered a teleoperator system, which includes the human operator (master) and a robot (slave). The operator controls the robot's movements and actions using her own movements (via special gear). A set of multiple sensors and displays give the operator sensory feedback (e.g. visual and tactile). As a result, the operator has the experience of being present in the same remote location as the robot. In some sense, the operator is being "incorporated" in the robot's body.

This idea of telepresence is often used in discussions concerning VR. However, in VR we do not have a remote location but a virtual one; we do not have a remotely controlled robot, but a virtual representation of a body (so-called "avatar"). A virtual environment is not somewhere "there", thus speaking about "being present there" is misleading. As we pointed out in section 1, a virtual environment is a representation or simulation of a physical space inhabited by the user's biological body via special VR gear. In short, a VR user is virtually present in a virtual environment.

So, what does it mean to be virtually present? Over the course of a two-decade debate, several definitions of virtual presence have been proposed (e.g. Slater \& Sanchez-Vives, 2005). Although all the definitions seem to share the belief that presence is a phenomenal state related to our experience, they differ in their scope and determinants. On one hand, we have experiential presence, which is defined very broadly as a mental state of being physically present as a result of perceptual stimulation (Draper et al. 1998). On the other, a definition of presence inspired by ecological and phenomenological approach states that it is "tantamount to successfully supported action in the environment" (Zahorik \& Jenison 1998). In the latter sense, the experience of presence is a result of performed actions in the environment. A similar definition was pro- 
posed by Biocca, according to whom presence is "the phenomenal state by which an individual feels located and active in an environment" (2001). We can see that in this sense multimodal stimuli, although necessary, is not a sufficient condition to develop the experience of presence. For this kind of presence, the essential factor is the sense of embodiment, i.e. sense of location, bodily agency and ownership (Kilteni et al. 2012) as well as the environment that affords actions.

In general, we can divide these conditions into technical (e.g. frames per second, virtual object realism, unlimited field of view etc.) and cognitive (e.g. possible interaction, sensorimotor contingencies etc.). As pointed out in section 1, we reserve the technical aspects for the notion of "immersion" and the psychological aspects for the notion of "presence". Following Sheridan (1992, 1996; Ijsselsteijn \& Reiner 2004), we can distinguish three main categories of determinants of virtual presence: sensory stimulation, user's level of control over interface mechanisms, and user's ability to modify the environment. Sensory stimulation refers to the amount and richness of sensory information provided by a VR interface. The second category concerns the "transparency" of a VR set and the perceptual possibilities it offers. However, it is also related to the correlation and congruence of a user's actions and movements with perceptual information. Sensorimotor congruence influences the ability to anticipate perceptual change, e.g. how the perceived object profile will change in accordance with a user's movement and predict what will happen. Finally, the third category introduces the key condition of developing the full experience of presence, namely a user's ability to modify the environment, i.e. to change and interact with perceived objects. We will argue that in order to interact with virtual objects the user has to first perceive possible actions that the object offers, so-called affordances (Gibson), as well as have a virtual representation of body.

The problem of measurement has appeared since VR researchers started to consider the experience of presence, which as a subjective state of experience is obviously difficult to measure. The degree of presence is usually measured by questionnaires. For instance, Witmer and Singer (1998) proposed a presence questionnaire based on distinguishing factors of control (e.g. degree of control), sensoriality (e.g. modality and consistency), distraction (e.g. VR interface awareness), and realism (e.g. scene realism). The questionnaire consists of several questions related to these factors in VR experience, e.g. "How natural did your interactions with the environment seem?", "How well could you examine objects from multiple viewpoints?”, and scale answers. The result of measurement can be used to determine how immersive a virtual environment is. 
Current VR systems are more complex and multimodal, thus it was proposed to use questionnaires in conjunction with other methods such as subjective reports and behavioral responses. For example, according to Kuschel et al. (2007) felt degree of presence is inversely proportional with degree of bimodal (e.g. visuotactile) incongruence. Thus, he proposes measuring multimodal conflicts as an indicator of deterioration of experience of presence. Another method focuses on reporting so-called breaks in presence (BIPs), i.e. specific experiential states of transition from the virtual to the real (Slater \& Steed 2000). For example, a VR user approaches a 3D object such as a tree and discovers that it has only a front side. In such moments, the feeling of virtual presence ("I am in a virtual garden") breaks down and the user awareness of the VR set (e.g. wearing a HMD), which was previously in the background, now becomes a main theme ("I am in a VR lab"). Finally, behavioral methods can be also applied in measuring presence in virtual reality. Observation of body posture, looming response, or measurement of galvanic skin response can indicate the degree of presence in the virtual environment as well as the sense of agency/ownership of the virtual body.

\section{Varieties of immersion}

Let us now move to the notion of "immersion". Even though this notion remains one of the key terms used both by academics and laymen interested in VR technologies, there is still surprisingly little agreement over a definition of it. Even a quick glance at the literature devoted to this notion shows that the main reason for this is that the common term "immersion" is simply too general to be of much use in academic contexts. Thus, most researchers focus on differentiating between various types (or aspects) of immersion. Interestingly, even though classifications of immersion are often developed with different aims in mind and result in different terminology, they end up with divisions that are structurally similar to each other. Let us look at two examples of such analysis of the notion of immersion and focus on similarity behind them.

The first example to be analyzed comes from (Ermi \& Mäyrä 2005). They propose dividing immersion into three types: challenge based, sensory, and imaginative. The way they characterize challenge-based immersion makes it very similar to the idea of "flow" famously introduced by (Csikszentmihályi 1990), as flow is often defined as a trance-like complete focus on the task. The result of this is that the task performer often forgets about her physical presence and the surrounding environment. Note that immersion in this sense rarely becomes associated with the notion of presence, as it is less about being transferred or transported to another place and more about losing the feeling of being in a particular place. You might say that the flow experience is much 
more about losing the feeling of presence in a given space than gaining a feeling of being somewhere else. ${ }^{7}$

The next type of immersion listed by (Ermi \& Mäyrä 2005) is sensory immersion. The main idea behind this notion is that it is supposed to be immersion resulting from perception of the environment by the user. This notion should be relatively easy to grasp, as it became the template for the popular image of technology-mediated immersion. Devices that induce immersion of this type are supposed to stimulate the user with artificial sensory stimuli that ideally become indistinguishable from the real thing. Note that even though the previous type of immersion (challenge based, flow-like immersion) also has to have a sensory component, the resulting psychological effect comes from focusing on the task and not on the vividness and plausibility of sensory experience. Contrary to this, sensory immersion can be easily produced in contexts that do not involve any particular task the user has to perform (for example in virtual tours or 360 degrees movies ${ }^{8}$ ). It is important to note that there is at least one important problem associated with this (idealized) picture. As shown in studies, the connection between the realism of sensory simulation (vividness, resolution etc.) and the sensory immersion it produces is much less obvious than it initially seems (Darken et al 1999, Dinh et al 1999, Zimmons \& Panter 2003). This initially seems rather surprising. Should the fidelity of a virtual environment not be directly proportionate to the feeling of presence? We will return to this problem in section 6.

The third type of immersion pointed out by Ermi and Mäyrä is "imaginative immersion". Despite the name, it is associated more with narratives than pictures. This type of immersion is interesting as it fits the earlier use of the word "immersion" (one that was coined well before anyone dreamed of VR techniques). Imaginative immersion is a state into which people enter whenever they are closely following a given narrative (reading a book, listening to a story, etc.). Although initially it may seem to be irrelevant for the problem of

\footnotetext{
7 It may be as well argued that challenge-based immersion offers temporal rather than spatial presence (of being present "in the moment" rather than "being there").

$8360^{\circ}$ movies are a relatively new form of cinema whose essential characteristic is that the field of view is so big that the viewer is completely surrounded, thereby creating a feeling of presence. Once you look at them from the perspective of affordances, they can be graded according to the level of perceptual freedom they offer the viewer. The easiest example is a movie that creates an impression of a projection on a circular screen (with the viewer in the center). The viewer has to rotate in order to look at everything displayed on the screen.I If we then enhance the movie by adding the third dimension, we give the user the ability to move in the $\mathrm{z}$ axis, meaning she can position herself closer and farther from some of the details on the screen. However, this can only be achieved with some type of tracking system that enables the software to measure depth of the movement and, at the same time, gives the viewer the ability to see parts of the movie from different perspectives. The most complex example of a $360^{\circ}$ movie is a movie that is rendered in real time. This technique gives the viewer the biggest number of affordances since she is not restricted to recorded camera movement and may change the perspective by focusing on any part of the movie.
} 
presence and immersion in VR, we will see that it is an important factor also in this modern context.

The second framework for immersion we wish to analyze is the aforementioned distinction proposed by Slater \& Wilbur (Slater \& Wilbur 1997). Although the authors talk about aspects of immersion (and not about its types), their classification can be easily matched with that of Ermi and Mäyrä. Slater and Wilbur point out that in order to achieve immersion the technology should be inclusive, extensive, surrounding, vivid, matching, and contain a plot (or a story). Let us quickly go through these requirements. The idea of "inclusiveness" boils down to the fact that immersive technology should be able to "shut down" the environment of the user, i.e. block (or downplay) the stimuli from her physical environment. The second requirement is that extensiveness pertains to the fact that immersive technology has to be multisensory (something that has been independently established by Leonardis et al. 2014). By stating that the technology also has to be surrounding and vivid, the authors allude to two very specific technical points: a simulation has to have a big field of view (the closer to $360^{\circ}$ the better) and the resolution of the picture and sound has to be up to a certain standard. The next requirement is that immersive technology has to be "matching", which simply means that it has to be synchronized well with a player's movement in order to simulate proprioception effectively. The two most important technical aspects this requirement results in are the framerate and the natural mapping ${ }^{9}$ of player and avatar movement.

The last of the aspects of immersive technologies (which is arguably the least technically oriented) is the plot requirement. The idea behind this requirement is rather simple: the narrative that accompanies the audio-visual experience should reinforce its cohesiveness.

Note that apart from one specific point (the matching requirement), the proposition of Slater \& Wilbur can be easily mapped into the account of Ermi and Mäyrä. The inclusiveness requirement can be interpreted as a flow-inducing aspect of the technology (thus, it directly relates to challenge-based immersion): the idea that immersive technologies have to be extensive, surrounding and vivid correlates with the idea of sensory immersion. Finally yet importantly, the plot requirement can be interpreted simply as imaginative immersion under a different name. However, what about the odd "matching" requirement? Can it be correlated with any of Ermi and Mäyrä's propositions or does it present a new, specific type of immersion?

\footnotetext{
${ }^{9}$ Although the idea of "natural mapping" has been used in the context of mimetic interfaces rather than VR (Skalski et al. 2011, Shafer \& Carbonara 2014), it is a rather good fit in this context. It simply means that the mapping between the body of the user and the body of the avatar is 1-1; therefore, there is no mediating layer of abstraction between the movement of the user and the movement of the avatar.
} 
We believe that once you stop looking at perception in terms of passive reception and employ a more modern Gibsonian or enactive (e.g. Noë 2004) perspective, this seemingly odd aspect of immersion fits nicely into the category of sensory immersion. The reason for this is that perception as conceived by enactive theories contains an active part in which the subject constantly probes the environment. This process would not work if it were not synchronized with subject movements and its position in space, etc. Thus, we believe that both models analyzed in this section are actually very much compatible. The only remaining task is to enhance the former by treating the sensory perception in enactive terms. Once you look at immersion from this point of view, the sensory aspect of it boils down to the right combination of perceptual affordances.

However, if we are to employ this enactive perspective on perception and look at VR from this point of view, we should first look more closely into how the key notions of ecological approach and enactivism (the notion of affordances) function in the context of virtual reality.

\section{Affordances}

The notion of affordances was introduced by James Gibson (e.g. 1977) and has since been applied and developed by numerous continuators of ecological psychology (e.g. Chemero 2003, Heft 1989, Turvey et al. 1981, Warren 1984). In the most general sense, "the affordances of the environment are what it offers the animal, what it provides or furnishes, either for good or ill", more specifically the affordance of something is "a specific combination of the properties of its substance and its surfaces taken with reference to an animal" (1977). Alternatively, to put it in other words, "affordance is the functional utility of an object for an animal with certain action capabilities" (Warren 1984). Concisely, affordances are dispositional properties of objects that are relative to the perceiver's body, sensorimotor abilities and current intention. Affordances are neither objective features of objects, such as shape, nor a subjective representation, but they are relational i.e. they emerge dynamically in a subject's perceptual and motoric activity in the environment. Moreover, the ecological approach to perception emphasizes the primacy of action, i.e. that we primarily perceive affordances of objects and secondarily perceive their features, such as shape or color.

Affordances can be measured. For example, Warren (1984) measured the "climbability" of stairs. In his research, he estimated the dependency between the perceiver's body (leg length, body mass) and the stairway (riser height, tread depth). As a result, he created a biomechanical model of stair climbing. Warren's research shows that the notion of affordance can be operationalized and used, for example, in the design of public spaces, environments and tools. 
Recent research shows that it is possible to measure perception of affordances in VR. For example, in Regia-Corte et al. (2013) participants were asked to estimate affordance of standing on a slanted surface perceived in virtual reality. Results confirmed that perception of affordances is possible in virtual environments and comparable with perception in real environments.

The split between psychology and technology we observed while discussing the difference between presence and immersion carries over to the notion of affordances. This is because you can easily look at affordances both from the experiencer's point of view (psychological point of view) and from the point of view of the designer of the experience (technological point of view).

Although the ecological approach and the notion of affordances are not new and appeared in VR research long ago (e.g. Smets et al. 1995; Flach \& Holden 1998; Zahorik \& Jenison 1998), they are still underestimated. Researchers who refer to Gibson's work focus mainly on the coupling of perception and action. Although sensorimotor dependencies are essentially important, a subject's activity in an environment cannot be fully understood without affordances and intentionality (spatiotemporal structure of action determined by its objective). We think that the concept of affordances together with the rich category of embodiment should be a central topic in the presence debate.

In some cases, affordances are taken into consideration in all but name, such as in the aforementioned (Sheridan 1992, 1996) model. As pointed out in section 2, Sheridan evaluates presence according to three criteria: (a) the fidelity of the multimodal displays; (b) the ability to modify sensor position; (c) and the ability to change the configuration of the environment. We propose combining $b$ and $c$ as they are simply two sides of the same coin, or two types of affordances we find in the environment. We think that fidelity could be in fact downplayed, as there are no doubts that it functions as a necessary condition because low fidelity displays lead to user discomfort. However, after a certain fidelity threshold (what this threshold is remains open to question) it stops being important and can perhaps even be detrimental to the feeling of presence, as highly realistic images may easily lead to high expectations of an environment's affordances (similar to the phenomenon of "uncanny valley").

To understand the role affordances play in creating and sustaining virtual reality, we have first to point out that the obvious prerequisite to being immersed or present in a virtual environment is to believe that there is some kind of alternative "place" in which we can immerse ourselves or be present (as opposed to a simple picture or an animation we can only look at from outside).

It is in fact deeply puzzling that virtual objects, environments or worlds can be so easily treated as "objects", "environments" and "worlds" respectively, and not merely as pictorial representations. When and why does a picture on 
screen become a virtual object or place? One obvious intuition would be that it has something to do with the move from a 2D to a 3D plane, but there has to be more to it than this. When a camera pans an object in a movie, people still treat the movie as an animation and not as a virtual world. It manifests itself in the obvious difference in verbal reports; the common descriptions of presence we mentioned in section 2 are typical ways users describe their experience of VR but they are far from typical when it comes to moviegoers. The key ingredient that is missing here is that the perception created in a movie is not active: perception has to be the action of a user to create the illusion of explorable space. Nowhere is this seen better than in the case of $360^{\circ}$ degree movies. ${ }^{10}$ Even though they lack interactivity and contain only a rudimentary set of perceptual affordances, they do create the feeling of presence (van den Boom et al. 2015). It is especially interesting that the feeling increases when the point of view of the user changes from observer to actor. This suggests that not only does the user have to believe in an explorable space, but also that the avatar she explores the space through remains embedded in this space as one of the objects in it.

This is how the notion of affordances helps explain the puzzling ontological shift in user perspective. Pictures become objects (environments or worlds respectively) once they start to be perceived as rather persistent, rich (possibly even infinite) sources of affordances. The simplest way to offer the required richness of affordances is to give the observer the possibility to be looked at from different sides. This effect can be achieved by letting users rotate the objects they encounter or freely move the camera. This movement can be shorter and less spectacular than a movie shot, but the fact that the user knows that it is just one of an infinite number of ways of observing the object creates the desired ontological shift.

\section{Virtual body - from rubber hand to full virtual body}

Since we have already mentioned the role of different points of view of the avatar the user embodies, it is only fitting to talk more about the idea of embodiment. According to Merleau-Ponty, embodiment is primarily experienced in our actions and perceptions (Merleau-Ponty 1945/2005). We know that we are embodied because we always perceive from a certain perspective and we move and actin an environment. Merleau-Ponty emphasizes that we cannot detach our body from the surrounding world or analyze the body in abstraction from the environment, as they are essentially interrelated. MerleauPonty's phenomenological approach is consistent with ecological and was an important source of inspiration for enactivism (e.g. Gallagher 2005, Noë 2004).

\footnotetext{
10 That is movies which enable the viewer to rotate the camera freely in $360^{\circ}$ (even though they remain linear, non-interactive experiences). They can be viewed without HMD's.
} 
It is interesting that there is controversy over the importance of embodiment to VR. Some argue that this technology can help humans to achieve a state of disembodiment (something mankind has always desired) (Balsamo 2000, Stone 2000, Ward 2001). Others try to prove the opposite; that embodiment remains a necessary condition and a facilitator of VR (Flichy 2007, Froy 2003, Hansen 2006, Mingers 2001). Others argue that it is crucial for the experience of presence in a virtual world (Slater et.al 2010b). We do not have the space to evaluate the discussion in question, but there seems to be at least one important conceptual confusion that should be pointed out. It seems that what most people argue about is how much the artificial body in VR has to be similar to your own (Normand, Giannopoulos, Spanlang, \& Slater, 2011, Petkova \& Ehrsson, 2008; Slater, Spanlang, Sanchez-Vives, \& Blanke, 2010a) and whether VR could be accessed without any body whatsoever. In a way, it is very hard to imagine (let alone design) a truly disembodied VR experience. This is because the moment you place a user avatar in a virtual space and give it even rudimentary ways to use the environment's affordances (for example, the ability to look at the environment from different perspectives), the user becomes embodied as she has to consider her own size and position in relation to observed objects. Even if the user does not have an avatar and is "embodied", from the camera's point of view he or she still would have a spatial orientation according to the camera's location. We can call this case a minimal virtual embodiment. Overall, we may conclude that a VR experience is always related to some degree of embodiment. Therefore, in the remaining part of the paper we focus on research that takes some form of embodiment in VR for granted. Recent discussions about the sense of embodiment in virtual reality concentrate on three topics: sense of self-location, sense of agency and sense of ownership (Kilteni et al. 2012). Most advanced studies concern inducing the sense of ownership of the virtual body.

The well-known rubber hand experiment shows how easily the feeling of ownership of one's own body can be extended onto an external object. The experiment is very simple: a participant sits with her hand lying on a desk but hidden from view. What she sees is a fake, rubber hand in front of her. The experimenter taps synchronously both the real and the fake hand. As a result, participants report that the location of the felt stimuli is moved to the location of the fake arm, which is usually interpreted as inducing a sense of ownership of the fake hand. The crucial determinant for this result is temporal congruence of visual and tactile information.

The same illusory effect can be induced using media or VR technology, i.e. when the rubber hand is replaced with a 3D image displayed on a screen or in an HMD (Slater 2009, Haans 2010). In different version of the experiment (Slater 2009, Tsakiris et al. 2006), tactile stimulation was replaced with proprioceptive feedback. Participants wore a data glove whose position was tracked by a computer, and an HMD in which they saw a virtual representation of the 
hand moving in synchrony with real hand movements. Again, the crucial factor was visual and, this time, motor synchrony. The participants felt as if the real hand was located in the position of the virtual hand, or as if the virtual hand were their own arm (Slater 2009).

Is it possible to induce a full body illusion? Firstly, we should consider the socalled "out of body experiments" performed by Olaf Blanke (2008). Participants wore HMD's to see their own body recorded by a camera behind them. Next, the experimenter stroked the participant's back, which the participant could see from distance in the HMD. Participants reported that after a while they felt as if they were looking at themselves from a distance with an ambiguous feeling of being between the first and third person perspectives. The experiment's design was modified by Petkova (2008) by replacing the observed body with a mannequin's body, or by a virtual representation of body. Subjects wore HMDs looking down at their belly; however, what they saw was the belly of a mannequin recorded from the point of view of a camera mounted on the mannequin's head (in other conditions the mannequin was replaced by a virtual representation of body perceived via HMD). Next, the researcher simultaneously stroked the bellies of the participant and mannequin, thereby inducing a sense of ownership of a perceived fake or virtual body.

Inducing a sense of agency has not been studied in as much detail as sense of ownership. So far, researchers have focused on measuring sense of agency as the subjective feeling of control of a virtual body. According to Kilteni et al. (2012), the sense of agency is sensitive to temporal discrepancies between proprioceptive and visual information. Thus, real-time mapping of this information is necessary to induce the feeling of control of one's virtual body. This is certainly a true but very limited account of bodily agency. Following the enactive approach, our everyday bodily experience of agency is not only related to our bodily movements, but also to our intentional actions and interactions with objects. We are agents as subjects of actions performed in an environment filled with tools and objects, which are means to achieve intended aims. The spatiotemporal structure of our actions is both constrained and regulated by the environment and an object's affordances. Therefore, a broader concept of sense of agency should consist of perception of affordances, adequate performance of action and realization of an action's objective. Including these three components of agency in VR research may help increase immersion and the experience of presence. 


\section{Conclusions}

Despite the multitude of approaches, theoretical models and vocabularies, a rather stable pattern emerges from the literature concerning the relation between the notions of immersion, presence and virtual reality. To put it in a very simplified manner, in order to achieve the feeling of presence, three requirements have to be met:

1. There is a minimal threshold of affordances which results in an ontological shift: the user starts to objectify the experience (treat it as an object, a place, a world, as opposed to an image or a movie).

2. There is a minimal degree of embodiment of the interface: the experience can be accessed via an interface which is objectified itself: it is perceived as being on the same ontological plane as the rest of the virtual environment (as opposed to the disembodied menu overlay mentioned in section 5, or a set of voice commands used off-screen).

3. The user embodies the interface via a reasonably natural mapping and perceives a virtual representation of his or her body. Natural mapping increases sense of ownership and agency.

We believe that VR technologies are so often identified with total immersion or presence because they usually meet these three minimal requirements by default. They meet requirement 1 by giving the user a number of perception affordances. By allowing the user to observe the environment (and its content) from a seemingly infinite number of perspectives they help to objectify the experience. They meet requirement 2 because for various reasons they do not use overlays but prefer to cast the user into the experience (even if only as a floating camera). ${ }^{11}$ They meet requirement 3 because by using HMD's they create a natural mapping between the movement needed to exploit perceptual affordances and the movement of the avatar (for example, instead of moving the camera with hands we move it with our head or eyes).

Requirements 1-3 offer answers to some of the questions associated with VR techniques. Firstly, they explain why the first person point of view creates a stronger sense of ownership (Petkova, Khoshnevis, \& Ehrsson, 2011; Slater, Spanlang, Sanchez-Vives, et al., 2010). This is because in the other case, the user has to embody a camera that is disconnected from the avatar the user is still controlling; therefore, the effect of 1-1 natural mapping breaks down. The experience of presence in such cases is disrupted by an ambiguous feeling of being somewhere between the first and third perspective, similar to results in out-of-body experiments (Blanke 2008).

\footnotetext{
11 Some of them are purely technological: for example, using a menu overlay in VR is inconvenient, as it demands the user to shift focus constantly.
} 
Secondly, they explain why graphics fidelity is not the most important aspect of immersive technologies, even though most of these techniques deal with sensory perception (second type of immersion in our classification). This is because the believability of perception comes from perceptual affordances and not from the sheer fidelity of pictures or sounds.

Thirdly, they show the limitations of the typical setup of head mounted displays, as they cannot deliver true 1-1 natural interface mapping. Most obviously, the movement of the avatar stops being immersive once it is mapped to something other than legs. For example, the movement of a thumb on a typical console controller or a movement of fingers on a keyboard.

Finally yet importantly, our proposal explains why experiences as simple as $360^{\circ}$ degree movies result in a feeling of presence; even though they are linear and not interactive, they allow for a minimal set of perceptual affordances (head movement) and embodiment.

Additionally, once you look at the phenomenon of presence from the perspective of requirements 1-3, it is quite easy to speculate on some future ways of intensifying it. There are two general strategies of achieving this. Firstly, we could focus on maximization of the sense of embodiment of the virtual body. The obvious strategy would be to assure a natural 1-1 mapping of user's body parts to those of the avatar. This can be achieved through a combination of haptic and mimetic interfaces. Note that recent popularity of the latter could be described as a reversal of the current rising popularity of VR technologies. Even though the bodies of users of popular consumer electronics of recent years (e.g. Wii, PlayStation Move, Microsoft Kinect) were more or less naturally mapped to the avatar's body, the effects of their actions were still observed from the outsider's perspective. The studies reviewed in sections 2 and 5 show that the desired effect of embodiment and ownership can also be enhanced by clever techniques similar those used in the rubber hand illusion experiments (they can be understood as forms of calibrations of natural 1-1 mapping between a user's body and the avatar). Moreover, increasing the sense of agency in a virtual environment requires further development of VR systems, especially haptic technology and dynamic scaling of affordances. The essential aspect of our bodily interaction with objects is tactile feedback and opportunities to manipulate. Tactile feedback is also important for regulating our behavior in order to complete a task. This can be provided by haptic technologies such as gloves or vests (e.g. Lindeman et al. 2004). Although this technology is still very young, we can certainly assert that it will increase the sense of embodiment in VR.

To understand the second strategy it is important to realize that-even though we claim that a certain minimal threshold of immersion is, in a way, guaranteed by VR techniques-you might still assume that adding affordances to the simulation will in fact strengthen the illusion. Another way is dynamic scaling 
of affordances. If affordances depend on individual body parameters (e.g. height, weight, limb length), then we need a mechanism for scaling environmental features and objects to each individual body in order to provide optimal perception. In this sense, a virtual environment should be personalized to actual user's body. This is necessary if we want users to achieve a specific task in a virtual environment, e.g. for training purposes. Perception of affordances and thus possibilities of actions should be dynamically adjusted to an individual's body.

Moreover, even though affordances responsible for the feeling of presence are mostly associated with the second type of immersion techniques (sensory immersion), both the other types of immersion can be used to mask deficiencies and lack of expected affordances. For example, a user could be given a pressing task to perform (challenge-based immersion) and shifts her attention only to elements of the environment crucial for the task (thus ignoring the lack of affordances in other places). On the other hand, the user could be presented with a narrative that explains the lack of expected affordances (for example, a user can be told that for some reason her body is immobilized). ${ }^{12}$

\section{References}

Balsamo, A. 2000. The virtual body in cyberspace. In: The cybercultures reader. D. Bell \& B. M. Kennedy, eds. Routledge, London and New York.

Biocca F. 2001. Inserting the Presence of Mind into a Philosophy of Presence: A Response to Sheridan and Mantovani and Riva. Presence, vol. 10: 554-556.

Blanke, O. Metzinger, T. 2008. Full-body illusions and minimal phenomenal selfhood. Trends In Cognitive Science, DOI: 10.1016/j.tics.2008.10.003.

Burge, T. 2005. Disjunctivism and Perceptual Psychology. Philosophical Topics, Vol. 33, No. 1: 1-78.

Chemero, A. 2003. An Outline of a Theory of Affordances. Ecological Psychology, 15(2): 181-195.

12 This role of perceptual affordances has recently been inadvertently confirmed by the new wave of consumer VR devices (the Oculus Vive, HTC Vive and PSVR). It seems that the biggest problem faced by developers is the discrepancy between the perceived movement of the player avatar and the movement of the player himself. The main reason that this causes problems is that it causes simulation sickness. Developers of software dedicated to these platforms try to solve this problem in several ways, but one that is especially relevant is that they purposefully restrict player movement by depicting situations in which the avatar sits, stands or walks in a very small perimeter that can be tracked by the VR equipment. Interestingly, the developers communicate these limits to the user via narrative immersion techniques. For example, one of the more successful games produced for modern platforms (Job Simulator) satirically depicts various tasks people perform while working in different environments. The fact that simulation depicts a titular "job" explains why the avatar cannot leave the place in which she is positioned (the player cannot abandon her workplace). 
Csikszentmihályi, M. 1990. Flow: The Psychology of Optimal Experience. Harper \& Row.

Darken, R. P. Bernatovich D. Lawson J. P. \& Peterson B. 1999. Quantitative Measures of Presence In: Virtual Environments: The Roles of Attention and Spatial Comprehension. Cyberpsychology and Behaviour 2(4): 337-347.

Dinh, H. Q. Walker N. Song C. Kobayashi A. \& Hodges L. F. 1999. Evaluating the Importance of Multi-sensory Input on Memory and the Sense of Presence In: Virtual Environments. Presented at IEEE Virtual Reality. Houston, Texas, US.

Draper, J.V., Kaber, D.B. \& Usher, J.M. 1998. Telepresence. Human Factors 40: 354-375.

Ermi, L., \& Mäyrä, F. 2005. Fundamental components of the gameplay experience: Analysing immersion. Worlds In: Play: International perspectives on digital games research, 37, 2.

Fiore S. M. et al. 2009. Virtual experiments and environmental policy. Journal of Environmental Economics and Management 57: 65-86.

Flach J. M., Holden J. G. 1998. The Reality of Experience: Gibsons Way. Presence, Vol. 7, No. 1: 90-95.

Flichy: 2007. The internet imaginaire. MIT Press, Cambridge.

Froy, F. 2003. Indeterminacy In: cyberspace. In: Indeterminate bodies. N. Segal, L. Taylor \& R. Cook, eds. Palgrave Macmillan, New York.

Gallagher, S. 2005. How the Body Shapes the Mind. Oxford Univ. Press.

Gibson J. J. 1997. The Theory of Affordances. In: Perceiving, Acting and Knowing. Shaw R., Bransford J. eds. New York: 67-82.

Haans A. 2010. The Embodied User. Corporeal Awareness \& Media Technology. Eindhoven Univ. of Technology.

Hall, T., W., Mojtaba N., Maslowski E., Petty, S. 2012). Virtual Reality as a Surrogate Sensory Environment, In: T. Gulrez, A. E. Hassanien, ed. Advances In: Robotics and Virtual Reality, Intelligent Systems Vol 26: 251-273.

Hansen, M. B. N. 2006. Bodies In: code: Interfaces with digital media. Routledge. New York.

Heft, H. 1989. Affordances and the Body: An Intentional Analysis of Gibson's Ecological Approach to Visual Perception, Journal for the Theory of Social Behaviour 19 (1).

IJsselsteijn W., Reiner M. 2004. On the importance of reliable real-time sensorimotor dependencies for establishing telepresence. In: Proceedings of Presence, Alcañiz Raya, M. \& Solaz B.R. eds.): 298-304.

Kilteni, K., Groten, R., \& Slater, M. 2012. The sense of embodiment In: virtual reality. Presence: Teleoperators and Virtual Environments, 21(4): 373-387.

Leonardis, D.; Frisoli, A.; Barsotti, M.; Carrozzino, M.; Bergamasco, M. 2014. Multisensory Feedback Can Enhance Embodiment Within an Enriched Virtual Walking Scenario, Presence: Teleoperators and Virtual Environments, vol. 23 (3): 253-2. 
Lindeman, R.W., Page, R., Yanagida, Y., Sibert, J.L. 2004. Towards Full-Body Haptic Feedback: The Design and Deployment of a Spatialized Vibrotactile Feedback System. Proc. of ACM Virtual Reality Software and Technology (VRST), Hong Kong, China: 146-149.

Lister, M. 2003. New media: A critical introduction. London: Routledge.

Merleau-Ponty M. 1945/2005. Phenomenology of Perception. Routledge.

Mingers, J. 2001. Embodying information systems: The contribution of phenomenology. Information and Organization, 11: 103-128.

Minsky M. 1980. Telepresence. OMNI.

Noë, A. 2004. Action in perception. MIT Press.

Normand, J. M., Giannopoulos, E., Spanlang, B., \& Slater, M. 2011. Multisensory stimulation can induce an illusion of larger belly size In: immersive virtual reality. PLoS One, 6 (1), e16128. doi:10.1371/journal.pone.0016128

Petkova V., Ehrsson H. 2008. If I Were You: Perceptual Illusion of Body Swapping, PLoS ONE 3 (12).

Petkova, V. I., Khoshnevis, M., \& Ehrsson, H. H. 2011. The perspective matters! Multisensory integration In: ego-centric reference frames determines full body ownership. Frontiers In: Psychology, 2, 35.

Sanchez-Vives, M. Slater, M. 2005. From Presence Towards Consciousness. Nature Reviews Neuroscience 6: 332-339.

Schroeder, R. 1996. Possible Worlds: The Social Dynamic of Virtual Reality Technologies. Boulder: Westview Press.

Schroeder, R. 2008. Defining Virtual Worlds and Virtual Environments. Journal of Virtual Worlds Research: Defining Virtual Worlds and Virtual Environments 2.

Shafer, D. M., Carbonara, C. P., (2014. Controller Required? The Impact of Natural Mapping on Interactivity, Realism, Presence and Enjoyment In: Motion-Based Video Games, Presence vol. 23 (3).

Sheridan, T. 1996. Further Musings on the Psychophysics of Presence. Presence Vol.5, No.2: 241-246.

Skalski, P., Tamborini, R., Shelton, A., Buncher, M., \& Lindmark: 2011. Mapping the road to fun: Natural video game controllers, presence, and game enjoyment. New Media and Society, 13 (2): 224-24.

Slater, M., Wilbur, S., (1997. A Framework for Immersive Virtual Environments (FIVE): Speculations on the Role of Presence In: Virtual Environments, Presence: Teleoperators and Virtual Environments, Vol. 6, No. 6: 603-616.

Slater M. et al. 2009. Inducing illusory ownership of a virtual body, Frontiers In Neuroscience 3(2).

Slater, M., Spanlang, B., Sanchez-Vives, M. V., \& Blanke, O. 2010a. First person experience of body transfer In: virtual reality. PLoS One, 5 (5). doi:10.1371/journal.pone.0010564 
Slater, M., Spanlang, B., \& Corominas, D.(2010b. Simulating virtual environments within virtual environments as the basis for a psychophysics of presence. ACM Transactions on Graphics, 29 (4), 92.

Slater, M. \& Steed, A. 2000. A virtual presence counter. Presence-Teleoperators and Virtual Environments 9: 413-434.

Smets G.J.F., Stappers, P.J. 1995. Designing In: virtual reality: perception-action coupling and affordances. In: Simulated and virtual realities: 189-208.

Steuer, J. 1992. Defining Virtual Reality: Dimensions Determining Telepresence, Journal of Communication 42(4): 73-93.

Regia-Corte T. et al. 2013. Perceiving affordances In: virtual reality: Influence of person and environmental properties In: perception of standing on virtual grounds, Virtual Reality Vol 17, No. 1: 17-28.

Sheridan T. B. 1992. Musings on telepresence and virtual presence, Presence: Teleoperators and Virtual Environments 1: 120-126.

Stone, A. R. 2000. Will the real body please stand up? Boundary stories about virtual cultures. In: The Cybercultures reader. D. Bell \& B. M. Kennedy, eds. Routledge, London and New York.

Turvey, M. T., Shaw, R.E., Reed, E., \& Mace, W. 1981). Ecological laws of perceiving and acting. In: reply to Fodor and Pylyshyn. Cognition, 9: 237-304.

Ward, K. 2001. Crossing cyber boundaries: Where is the body located In: the online community? In: Reframing the body. N. Watson and S. Cunningham-Burley, eds. Palgrave, NY.

Warren William H. Jr.(1984. Perceiving Affordances: Visual Guidance of Stair Oimbing, Journal of Experimental Psychology: Human Perception and Performance, vol. 10 (5). pp. 683-703.

van den Boom, A. A., Stupar-Rutenfrans, S., Bastiaens, O. S., \& van Gisbergen, M. M. 2015). Observe or Participate: The Effect of Point-Of-View on Presence and Enjoyment In: 360 Degree Movies for Head Mounted Displays. Proceedings of Workshop and Poster Papers of the European Conference on Ambient Intelligence 2015 (AmI15), Athens, Greece, November: 11-13.

Witmer, B. G., \& Singer, M. J. 1998. Measuring presence in virtual environments: A presence questionnaire. Presence: Teleoperators and Virtual Environments, 7(3): 225-240.

Zahorik: \& Jenison, R.L. 1998. Presence as being-in-the-world. Presence. Teleoperators and Virtual Environments 7: 78-89.

Zimmons: \& Panter A. 2003. The Influence of Rendering Quality on Presence and Task Performance In: a Virtual Environment. Proceedings of the IEEE Virtual Reality. 